\title{
Risk Factors for Chikungunya Virus Outbreak in Somali Region of Ethiopia, 2019: Unmatched Case-Control Study
}

\author{
Mikias Alayu (D), ${ }^{1}$ Tesfalem Teshome, ${ }^{2}$ Hiwot Amare, ${ }^{1}$ Solomon Kinde, ${ }^{1}$ Desalegn Belay, ${ }^{1}$ \\ and Zewdu Assefa ${ }^{1}$ \\ ${ }^{1}$ Ethiopian Public Health Institute, Center for Public Health Emergency Management, Addis Ababa, Ethiopia \\ ${ }^{2}$ St. Paul's Hospital Millennium Medical College, Department of Public Health, Addis Ababa, Ethiopia \\ Correspondence should be addressed to Mikias Alayu; mikiasalayu7@gmail.com
}

Received 14 August 2020; Revised 9 January 2021; Accepted 2 February 2021; Published 10 February 2021

Academic Editor: Jay C. Brown

Copyright $\odot 2021$ Mikias Alayu et al. This is an open access article distributed under the Creative Commons Attribution License, which permits unrestricted use, distribution, and reproduction in any medium, provided the original work is properly cited.

\begin{abstract}
Background. Chikungunya virus is a ribonucleic acid (RNA) virus transmitted by a mosquito bite. Chikungunya virus outbreaks are characterized by rapid spread, and the disease manifests as acute fever. This study aimed at determining risk factors for chikungunya virus outbreak to apply appropriate prevention and control measures. Methods. Unmatched case-control study was performed to identify risk factors of chikungunya outbreak in Somali region of Ethiopia in 2019. Cases and controls were enrolled with $1: 2$ ratio. All cases during the study period ( 74 cases) and 148 controls were included in the study. Bivariate and multivariable analyses were implemented. The serum samples were tested by real-time polymerase chain reaction at Ethiopian Public Health Institute Laboratory. Results. A total of 74 chikungunya fever cases were reported starting from $19^{\text {th }}$ May 2019 to $8^{\text {th }}$ June 2019 . Not using bed net at daytime sleeping (adjusted odds ratio (AOR): 20.8; 95\% confidence interval (CI): 6.4-66.7), presence of open water holding container (AOR: 4.0; CI: 1.2-3.5), presence of larvae in water holding container (AOR: 4.8; CI: 1.4-16.8), ill person with similar signs and symptoms in the family or neighbors (AOR: 27.9; CI: 6.5-120.4), and not wearing full body cover clothes (AOR: 8.1; CI: 2.2-30.1) were significant risk factors. Conclusion. Not using bed net at daytime sleeping, presence of open water holding container, presence of larvae in water holding container, ill person with similar signs and symptoms in the family or neighbors, and not wearing full body cover clothes are risk factors for chikungunya virus outbreak.
\end{abstract}

\section{Background}

Chikungunya virus (CHIKV) is a ribonucleic acid (RNA) virus that belongs to the Alphavirus genus of the Togaviridae family transmitted by the bite of mosquitoes Aedes aegypti and Aedes albopictus. CHIKV outbreaks are characterized by rapid spread and infection rates as high as $75 \% ; 72 \%-93 \%$ of infected persons become symptomatic. The disease manifests as acute fever and potentially debilitating polyarthralgia [1].

The Aedes mosquitoes breed in domestic settings such as flower vases and water storage containers and peridomestic areas such as construction sites, coconut shells, and discarded household junk items (vehicular tires, plastic cans, and metal cans). Adult mosquitoes rest in cool and shady areas in domestic and peridomestic settings and bite humans commonly during the daytime [2].

Since the first outbreak in Tanzania in 1952, chikungunya virus has caused outbreaks in various parts of Africa. Chikungunya virus has been found to circulate in Eastern and Central Africa [3]. Chikungunya fever is commonly a self-resolved disease. Whereas, patients with coexisting conditions such as cardiovascular, neurologic, and respiratory disorders or diabetes need hospitalization. Additionally, chikungunya virus may present with bleeding when coexisting with dengue fever [4-6].

Chikungunya virus is a highly contagious disease that can affect up to $70 \%$ of the total population of the outbreak affected area. The virus can easily transmit across continents and the current growing movement of people from one 
country to another country as well as international trade facilitates the importation of the virus [7].

Typical presentations of chikungunya virus infection are sudden onset of fever and joint pain, but sometimes, it may cause severe complications including myocarditis, meningitis, encephalitis, and flaccid paralysis [8].

Frequent outbreaks of chikungunya virus infection suggest that health system efforts for vector control alone may not be sufficient for effective control. A combination of health system efforts and healthy behavior practices by the community is essential for effective control of chikungunya outbreak [9].

The prevention mechanisms for chikungunya virus are to reduce human mosquito contact or eliminate vector populations. In this regard, control measures should be focused on eliminating the immature stages of the mosquitoes and their larval developmental sites [10].

This study was conducted to determine risk factors for the chikungunya outbreak in Somali region of Ethiopia to implement targeted prevention and control measures.

\section{Methods}

2.1. Study Area and Period. This study was conducted at Kebridahar City Administration of Somali region. Kebridahar city is located 1006 kilometers to the east direction of Addis Ababa and 380 kilometers away from Jigjiga, the capital city of Somali region. The city administration has ten kebeles. The area is lowland with temperature ranging from $32^{\circ} \mathrm{C}$ to $40^{\circ} \mathrm{C}$. According to projection of the 2007 census of Ethiopia, the city administration has a total population of 117,222 . Fifty-seven percent $(66,817)$ of them were males and $12 \%(14,067)$ was children below five years of age [11]. There were two public hospitals in Kebridahar City Administration. This outbreak investigation was conducted for one month, from $25^{\text {th }}$ May 2019 to $25^{\text {th }}$ June 2019.

2.2. Study Design. Unmatched case-control study was conducted to determine the risk factors of chikungunya fever disease outbreak in Kebridahar City Administration of Somali region.

2.3. Source Population. All the residents of Kebridahar city were the source population.

2.4. Study Population. The study populations for cases were people who fulfill the case definition of chikungunya virus. The study populations for controls were people who did not have chikungunya virus disease. Individuals with any kind of severe illness and mental disorders were excluded from the study.

2.5. Sampling Method and Sample Size. For this study, the case to control ratio was 1 to 2 . All cases and two controls for each case were included. Therefore, 74 cases and 148 controls participated in the study.
2.6. Selection of Cases. All individuals who fulfill the case definition of chikungunya fever and who were willing to participate in the study were included in the study as a case. The cases were identified both from the health facilities and from the community by active case search.

2.7. Selection of Controls. Two controls for each case were selected from the neighbor households. One control was taken randomly by the lottery method from members of the household on the right side of the case's house, and the other control was selected by the same method from the household on the left side of the case's house.

\section{Case Definitions and Outbreak Threshold}

3.1. Confirmed Case. A suspected case with one of the following laboratory findings.

(i) Isolation of virus or demonstration of specific viral antigen or nucleic acid in tissue, blood, or other body fluid, OR

(ii) Four-fold or greater change in virus-specific quantitative antibody titers in paired serum samples, OR

(iii) Virus-specific IgM antibodies in serum with confirmatory neutralizing antibodies in the same or later specimen [12]

3.2. Outbreak Threshold. In the nonendemic area, a single case of suspected chikungunya virus is considered as a suspected outbreak, and if one case is confirmed by one of the laboratory methods, it is considered as a confirmed outbreak [13].

\section{Operational Definitions}

Breteau index: number of containers which have larvae of Aedes mosquito per 100 households inspected.

House index: percentage of households where larvae of Aedes mosquito were identified per number of households inspected.

Container index: percentage of containers that have at least one larvae or pupa of Aedes mosquito per number of containers inspected.

Positive household for Aedes mosquito: a household in which at least one larvae or pupa of Aedes mosquito was identified in at least one water container.

Negative household for Aedes mosquito: a household in which no larvae or pupa of Aedes mosquito was identified.

Positive container for Aedes mosquito: a water holding container in which at least one larvae or pupa of Aedes mosquito was found.

Negative container for Aedes mosquito: a water holding container in which larvae or pupas of Aedes mosquito were not found.

Kebele: the lowest political administration structure in the Ethiopian administration system. 
Epidemiologically linked: cases who have been living in the same local area with confirmed cases for fourteen days prior to the date of onset of fever and/or joint pain.

4.1. Data Collection. Epidemiological data were collected by face-to-face interview of cases and controls. The data collection tool was originally prepared in English language, and it was translated to Somali. The tool contains sociodemographic information, current clinical information, and risk factor information. Data collectors were health officers and nurses who can speak Somali. One day training was given to data collectors.

Entomological data were collected by observation of water containers among selected households from four high cases reporting kebeles (02, 03,09, and 10). The larvae and pupas were collected by dipper and pipette and put them in a well-labeled cup with net cover to allow them to grow into adult mosquitoes. Once the adult mosquitos were grown, Aedes mosquito was identified by mosquito identification key.

Regarding the human (laboratory) sample for confirmation, five serum samples were collected from suspected cases and transported to Ethiopian Public Health Institute (EPHI) Arbovirus Laboratory as per the recommended cold chain protocol. The laboratory expert from the hospital transported the sample to EPHI as per the recommended cold chain protocol.

4.2. Data Analysis and Presentation. After the data were cleaned and checked for completeness, entered into Epi Info Version 7.2 and exported to Statistical Package for Social Science (SPSS) version 23. Descriptive analyses by person, place, and time were performed. Bivariate and multivariable binary logistic regressions were performed to identify risk factors for this chikungunya outbreak. The result was interpreted by using odds ratio with $95 \%$ confidence level and $p$ value of 0.05 .

Breteau index, house index, and container index were calculated for entomological data.

To confirm the etiologic agent of the outbreak, serum samples were tested by real-time polymerase chain reaction (RT-PCR) after viral ribose nucleic acid (RNA) was extracted.

4.3. Ethical Consideration. A support letter was written by Ethiopian Public Health Institute, Center for Public Health Emergency Management (PHEM), to Somali Regional Health Bureau and Kebridahar city Administration Health Office. Permission to investigate the outbreak was obtained from the Regional Health Bureau and the City Health Office as well as the mayor office of Kebridahar City Administration. The ethical review was exempted as it was outbreak investigation and response endeavor.

The interviewers had explained about the objectives, the process, and the benefits of the study for each participant. Each participant was asked for their informed consent, and interview was conducted after written consent was received from the participant. In case of interviewing children, the consent was obtained from their parents and guardians. Cases identified during data collection were sent to a health facility for treatment.

\section{Result}

5.1. Sociodemographic Characteristics of Respondents. A total of 222 participants, 74 cases and 148 controls, were interviewed for this study with $100 \%$ response rate.

From the total participants, 59\% (132) was female, 14\% (32) was children below five years old, 58\% (129) has no formal education, 59\% (131) was married, 41\% (92) was house wives, and $13 \%$ (29) has more than ten family members (Table 1).

5.2. Description of Cases. A total of 74 chikungunya fever cases were reported from Kebridahar City Administration starting from $19^{\text {th }}$ May 2019 to $8^{\text {th }}$ June 2019 (Figure 1).

Eighty-nine percent (66) were treated as an outpatient and $10.8 \%(12 / 74)$ cases were treated as an inpatient.

Among a total of 74 cases, 41 (55.4\%) were males and 33 (44.6\%) were females. Two cases were children less than five years old and seven cases were above 45 years old. The overall attack rate of the outbreak was 63 cases per 100,000 at-risk population. The highest attack rate was among the age group of $15-44(83 / 100,000)$ and the lowest attack rate was among the age group of below five years $(22 / 100,000)$ (Table 2). The median age of cases was 25 years (IQR: 20-33).

The case fatality rate of this outbreak was zero. The attack rates among males and females were 62 and 66 cases per 100,000 risk population, respectively.

5.3. Signs and Symptoms. All cases had fever and joint pain. None of the cases had bleeding (Table 3).

5.4. Entomological Findings. A total of 26 households and 49 water containers were visited from four kebeles of Kebridahar city to identify the mosquito species. Among those containers in the visited households, 26.5\% (13/49) was positive containers and the rest $73.5 \%(36 / 49)$ was negative containers.

Of the visited households, 38.5\% (10/26) was positive households. The highest Breteau index and house index were identified from kebele ten, whereas the highest container index was in kebele two of the city (Table 4).

5.5. Risk Factors of Chikungunya Virus Outbreak. In multivariable analysis, eight variables significant by bivariate analysis were entered in the model. The odds of being affected by chikungunya disease was 21 times higher among people not using bed net at daytime sleeping (adjusted odds ratio (AOR): 20.8; 95\% confidence interval (CI): 6.4-66.7), four times higher among people who have open water containers (AOR: 4; CI:1.2-13.5), five times higher among people whose water container had mosquito larvae (AOR: 4.8; CI: 1.4-16.8), 28 times higher among people who have 
TABLE 1: Sociodemographic characteristics of study participants, Kebridahar City Administration, Korahe zone of Somali region, Ethiopia, 2019.

\begin{tabular}{|c|c|c|c|c|c|}
\hline \multirow{2}{*}{\multicolumn{2}{|c|}{ Variables and categories }} & \multicolumn{2}{|c|}{ Cases } & \multicolumn{2}{|c|}{ Controls } \\
\hline & & Number & Percentage (\%) & Number & Percentage (\%) \\
\hline \multirow{2}{*}{ Sex } & Female & 33 & 45 & 95 & 64 \\
\hline & Male & 41 & 55 & 53 & 36 \\
\hline \multirow{4}{*}{ Age (in years) } & Under 5 & 2 & 3 & 30 & 20 \\
\hline & $5-14$ & 16 & 22 & 33 & 22 \\
\hline & $15-45$ & 49 & 66 & 54 & 36 \\
\hline & Above 45 & 7 & 9 & 31 & 21 \\
\hline \multirow{4}{*}{ Educational status } & No formal education & 51 & 69 & 78 & 53 \\
\hline & Below school age & 1 & 1 & 12 & 8 \\
\hline & Primary school & 15 & 20 & 43 & 29 \\
\hline & Secondary school & 7 & 9 & 15 & 10 \\
\hline \multirow{4}{*}{ Marital status } & Married & 56 & 76 & 75 & 51 \\
\hline & Single & 0 & 0 & 15 & 10 \\
\hline & Widowed & 0 & 0 & 11 & 7 \\
\hline & Under age & 18 & 24 & 47 & 32 \\
\hline \multirow{5}{*}{ Occupation } & Daily laborer & 11 & 15 & 0 & 0 \\
\hline & House wife & 24 & 32 & 68 & 46 \\
\hline & Merchant & 21 & 28 & 22 & 15 \\
\hline & Student & 18 & 24 & 46 & 31 \\
\hline & Others & 0 & 0 & 12 & 8 \\
\hline \multirow{3}{*}{ Family size } & Less than 5 & 25 & 34 & 49 & 33 \\
\hline & $5-10$ & 40 & 54 & 79 & 53 \\
\hline & Above 10 & 9 & 12 & 20 & 14 \\
\hline
\end{tabular}



Date of onset of fever

Figure 1: Distribution of chikungunya virus cases by date of onset of fever in Kebridahar City Administration, Somali, Ethiopia, 2019.

TABle 2: Distribution of chikungunya cases by the age group, Kebridahar City Administration, Somali, Ethiopia, 2019.

\begin{tabular}{|c|c|c|c|c|c|}
\hline \multicolumn{2}{|c|}{ Variables and categories } & Number of cases & Percentage $(\%)$ & At-risk population & Attack rate per 100,000 at-risk population \\
\hline \multirow{4}{*}{ Age (in years) } & Under 5 & 2 & 2.7 & 9,050 & 22 \\
\hline & $5-14$ & 16 & 21.6 & 35,970 & 44 \\
\hline & $15-44$ & 49 & 66.2 & 59,047 & 83 \\
\hline & Above 45 & 7 & 9.5 & 13,155 & 53 \\
\hline \multirow{2}{*}{ Sex } & Male & 41 & 55.4 & 66,817 & 62 \\
\hline & Female & 33 & 44.6 & 50,405 & 66 \\
\hline \multicolumn{2}{|l|}{ Total } & 74 & 100.00 & 117,222 & 63 \\
\hline
\end{tabular}


Table 3: Distribution of chikungunya cases by sign and symptoms in Kebridahar City Administration, Somali, Ethiopia, 2019.

\begin{tabular}{lcccc}
\hline Sign and symptom & Yes (number) & Percentage (\%) & No (number) & Percentage (\%) \\
\hline Fever & 74 & 100 & 0 & 0 \\
Joint pain & 74 & 100 & 21 & 0 \\
Headache & 53 & 72 & 66 & 28 \\
Rash & 8 & 11 & 37 & 89 \\
Nausea/vomiting & 37 & 50 & 74 & 50 \\
Bleeding & 0 & 0 & & 100 \\
\hline
\end{tabular}

Table 4: Indices of Aedes aegypti mosquito by kebele in Kebridahar City Administration, Somali, Ethiopia, 2019.

\begin{tabular}{llcccc}
\hline \multirow{2}{*}{$\begin{array}{l}\text { Location investigated } \\
\text { District }\end{array}$} & Kebeles & Type of vector found & \multicolumn{3}{c}{ Aedes aegypti Stegomyia indices } \\
& 02 & Aedes aegypti & 50 & 37.5 & Container index (\%) \\
\hline \multirow{3}{*}{ Kebridahar city } & 03 & Aedes aegypti & 28.5 & 28.5 & 50 \\
& 09 & Aedes aegypti & 33.3 & 33.3 & 22.2 \\
& 10 & Aedes aegypti & 66.6 & 44.4 & 28.6 \\
\hline
\end{tabular}

neighbors with chikungunya virus signs and symptoms (AOR: 27.9; CI: 6.5-120.4), and eight times higher among people who usually wears cloths that do not cover full body (AOR: 8.1; CI: 2.2-30.1) (Table 5).

\section{Discussion}

CHIKV has caused numerous epidemics around the world, including new spread to previously nonendemic regions, such as the America, Europe, the Middle East, and Oceania [14]. The first outbreak in Ethiopia was detected in Dolo Ado district of Somali region in 2016; since then, this is the third outbreak following a similar outbreak in Adar district of Afar region in March 2019.

Among the total cases, $10.8 \%$ was severe and needs admission, which is lower than the similar study in Malaysia. That study also showed that the attack rate ranged from 0.6 to 63 per 100,000 population in different districts [15]. In our study, the overall attack rate of the outbreak was 63 cases per 100,000 at-risk population. The case fatality rate of the chikungunya outbreak in Kebridahar city is zero. Similarly, studies in Malaysia showed that the case fatality rate of chikungunya virus is zero $[3,15]$.

The median age of the chikungunya fever cases in Kebridahar city was 25 years; this finding is almost similar to the median age of chikungunya fever cases (24 years) found from a cross-sectional study in Tanzania [16].

Our investigation results also showed that all cases had fever and joint pain, but no case was presented with bleeding. This could be because of the acute phase of chikungunya virus infection characterized by high grade fever and severe joint pain, and bleeding is less likely among individuals infected by chikungunya virus [17].

The finding of our study showed that the Breteau index and house index of Aedes aegypti ranged from $28.5 \%$ to $66.6 \%$ and from $28.5 \%$ to $44.4 \%$, respectively. Aedes mosquito larval index is categorized as high and low larval indices based on house and Breteau indices. High larval index is when the house index is $\geq 5 \%$ and/or Breteau index is $\geq 20 \%$ [18].
In this outbreak investigation, the odds of developing chikungunya virus disease is 21 times higher among peoples who did not use bed net during daytime sleep compared to those used and the odds of developing chikungunya virus disease is eight times higher among peoples wearing shorts and T-shirts than peoples wear full body cover closes. In support of these findings, a study by Ramachandran et al. identified that not wearing clothes that fully cover the body is a risk factor for CHIKV infections and another study by Degifie et al. indicated not using bed net is a risk factor for dengue fever which shares a common vector with CHIKV for transmission $[19,20]$.

This investigation also revealed that the odds of developing chikungunya virus disease is four times higher among peoples having open water holding containers compared to those who properly close their containers and four points eight times higher among peoples whose water container had larvae of mosquitos compared to those whose water containers did not have larvae. A cross-sectional study in Tanzania also indicates that the seroprevalence of chikungunya virus is higher among participants with uncovered water container [16]. Another study in Indonesia also indicated that poor practices in eliminating mosquito breeding sites are risk factors for chikungunya virus. This supports the findings of our study that people whose water container had larvae of mosquitos is a risk factor for chikungunya virus disease [21].

Our study indicated that the risk of developing chikungunya virus disease is higher among people who are having neighbors and family members manifesting signs and symptoms of chikungunya virus. This finding is supported by a population-based cross-sectional study in the Reunion Island [22].

On the other hand, a retrospective study of chikungunya outbreak in India found that differences in awareness of chikungunya, cause of the disease, vector responsible, mode of transmission, biting time, and elimination of breeding of mosquitoes are significant risk factors, which are not significantly associated with chikungunya virus infection in our study [4]. 
Table 5: Multivariable analysis of chikungunya risk factors in Kebridahar City Administration of Somali, Ethiopia, 2019.

\begin{tabular}{|c|c|c|c|c|c|}
\hline Variables and categories & Cases $(\%)$ & Controls (\%) & COR $(95 \% \mathrm{CI})$ & AOR (95\% CI) & $p$ value \\
\hline \multicolumn{6}{|l|}{ Sex } \\
\hline Female & $33(44.6)$ & $95(64.2)$ & 1 & 1 & \multirow{2}{*}{0.331} \\
\hline Male & $41(55.4)$ & $53(35.8)$ & $1.8(1.1-3.2)$ & $1.8(0.6-6.1)$ & \\
\hline \multicolumn{6}{|c|}{ Knowing symptoms and preventions of chikungunya } \\
\hline Yes & $13(17.6)$ & $71(48.0)$ & 1 & 1 & \multirow{2}{*}{0.075} \\
\hline No & $61(82.4)$ & $77(52.0)$ & $4.3(2.2-8.5)$ & $3.3(0.9-12.0)$ & \\
\hline \multicolumn{6}{|c|}{ Knowing that Aedes mosquito bites commonly at daytime } \\
\hline Yes & $21(28.4)$ & $65(43.9)$ & 1 & 1 & \multirow{2}{*}{0.189} \\
\hline No & $53(71.6)$ & $83(56.1)$ & $1.9(1.1-3.6)$ & $2.2(0.7-7.0)$ & \\
\hline \multicolumn{6}{|c|}{ Using bed net at daytime sleeping } \\
\hline Yes & $12(16.2)$ & $130(87.8)$ & 1 & 1 & \multirow{2}{*}{$<0.001^{*}$} \\
\hline No & $62(83.8)$ & $18(12.2)$ & $37.3(16.9-82.3)$ & $20.8(6.4-66.7)$ & \\
\hline \multicolumn{6}{|c|}{ Status of water holding container } \\
\hline Closed & $23(31.1)$ & $123(83.1)$ & 1 & 1 & \multirow{2}{*}{$0.023^{*}$} \\
\hline Open & $51(68.9)$ & $25(16.9)$ & $10.9(5.7-20.9)$ & $4.0(1.2-13.5)$ & \\
\hline \multicolumn{6}{|c|}{ Presence of larvae in water holding container } \\
\hline Yes & $50(67.6)$ & $21(14.2)$ & $12.6(6.4-24.6)$ & $4.8(1.4-16.8)$ & \multirow{2}{*}{$0.015^{*}$} \\
\hline No & $24(32.4)$ & $127(85.8)$ & 1 & 1 & \\
\hline \multicolumn{6}{|c|}{ Ill person with chikungunya in the house hold and neighbors } \\
\hline Yes & $47(63.5)$ & $15(10.1)$ & $21.7(9.3-50.7)$ & $27.9(6.5-120.4)$ & \multirow{2}{*}{$<0.001^{*}$} \\
\hline No & $27(36.5)$ & $133(89.8)$ & 1 & 1 & \\
\hline \multicolumn{6}{|c|}{ Kind of clothes they wear commonly since the emergence of this outbreak } \\
\hline Full body cover & $22(29.7)$ & $115(77.7)$ & 1 & 1 & \multirow{2}{*}{$0.002^{*}$} \\
\hline Short & $52(70.3)$ & $33(22.3)$ & $8.2(4.4-15.5)$ & $8.1(2.2-30.1)$ & \\
\hline
\end{tabular}

COR, crude odds ratio; AOR, adjusted odds ratio; CI, confidence interval. * $p$ value less than 0.05 .

In a case-control study, the informations were collected retrospectively. Therefore, the trueness of the information depends on the recalling ability of the participants. The other limitation of this study could be asymptomatic chikungunya virus cases might not be detected.

\section{Conclusion}

This outbreak was the third chikungunya outbreak in Ethiopian by which no death was registered.

Not using bed net during daytime sleeping, having an open water container, presence of Aedes mosquito larvae in water holding container, living with people having chikungunya signs and symptoms, and wearing clothes which did not cover the full body were risk factors for being affected by chikungunya fever.

Therefore, there should be regular indoor and outdoor spraying of insecticidal chemicals, regular monitoring of water containers which are difficult to drain as well as to cover and apply larvicidal chemicals, awareness creation on daytime bed net utilization, drainage of unusable stored water, educating the community to cover all water containers in and around the house, promoting people to wear full body cover cloths, and using mosquito repellants in the time when there is an outbreak are helpful to prevent and control chikungunya outbreaks.

\section{Data Availability}

The data used to support the findings of this study are available from the corresponding author upon request.

\section{Conflicts of Interest}

The authors declare that they have no conflicts of interest.

\section{Authors' Contributions}

MA designed the study, participated in field investigation, conducted analysis, and wrote the manuscript. TT participated in designing the study and manuscript writing. HA and DB involved in laboratory confirmation. HA and SD participated in field investigation and data analysis. All authors reviewed the manuscript and approved the submission.

\section{Acknowledgments}

The authors would like to acknowledge Ethiopian Public Health Institute and Somali Regional Health Bureau for facilitating the investigation and financial support during the investigation time.

\section{References}

[1] R. S. Nasci, "Movement of chikungunya virus into the western hemisphere," Emerging Infectious Diseases, vol. 20, no. 8, pp. 1394-1395, 2014.

[2] World Health Organization Regional Office for South East Asia, Guideline for Prevention and Control of Chikungunya Fever, http://www.wpro.who.int/mvp/topics/ntd/ Chikungunya_WHO_SEARO.pdfAccessed 6 July, 2019.

[3] M. C. Williams, J. P. Woodall, P. S. Corbet, and J. D. Gillett, "O'nyong-nyong fever: an epidemic virus disease in East 
Africa VIII. Virus isolations from anopheles mosquitoes," Transactions of the Royal Society of Tropical Medicine and Hygiene, vol. 59, no. 3, pp. 300-306, 1965.

[4] B. N. Nagpal, R. Saxena, A. Srivastava et al., "Retrospective study of chikungunya outbreak in urban areas of India," Indian Journal of Medical Research, vol. 135, no. 3, pp. 351-358, 2012.

[5] S. C. Weaver and M. Lecuit, "Chikungunya virus and the global spread of a mosquito-borne disease," New England Journal of Medicine, vol. 372, no. 13, pp. 1231-1239, 2015.

[6] J. E. Staples, R. F. Breiman, A. M. Powers, and C. Fever, "Chikungunya fever: an epidemiological review of a Reemerging infectious disease," Clinical Infectious Diseases, vol. 49, no. 6, pp. 942-948, 2009.

[7] F. N. Rodrigues, J. Lourenço, C. E. Marques, L. M. Maia, O. Pybus, and A. L. Carlos, "Epidemiology of chikungunya virus in bahia, Brazil, 2014 - 2015," PLOS Current Outbreaks, vol. 1-9, 2016.

[8] H. Appassakij, P. Khuntikij, M. Kemapunmanus, R. Wutthanarungsan, and K. Silpapojakul, "Viremic profiles in asymptomatic and symptomatic chikungunya fever: a blood transfusion threat?" Transfusion, vol. 53, no. 10pt2, pp. 2567-2574, 2013.

[9] V. Ramachandran, P. Manickam, P. Kaur et al., "Behavioural determinants associated with CHIKV outbreak in gouriepet, avadi, Chennai, south India," Journal of Biomedical Science, vol. 4, no. 1, pp. 1-6, 2016.

[10] M. Dhimal, I. Gautam, H. D. Joshi, R. B. O’Hara, B. Ahrens, and U. Kuch, "Risk sactors for the presence of Chikungunya and Dengue Vectors (Aedes aegypti and Aedes albopictus), Their altitudinal distribution and climatic determinants of their abundance in Central Nepal," PLOS Neglected Tropical Diseases, vol. 9, no. 3, pp. 1-20, 2015.

[11] Central Statistics Agency of Ethiopia, "Projected population of Ethiopia," 2019, https://www.statsethiopia.gov.et/ population-projection/Accessed on 14th December, 2020.

[12] M. S. Hossain, M. M. Hasan, M. S. Islam, S. Islam, M. Mozaffor et al., "Chikungunya outbreak in Bangladesh: clinical profile, economic impact and quality of life during the acute phase of the disease," PLOS Neglected Tropical Diseases, vol. 12, no. 6, pp. 1-16, 2018.

[13] Ethiopian Public Health Institute, "Public health emergency management guideline for Ethiopia," https://www.ephi.gov. et/images/guidelines/phem-guideline-final.pdfAccessed on 6 July, 2019.

[14] I. Sam, B. Kümmerer, F. Chan, P. Roques, and A. Christian, "Updates on Chikungunya epidemiology, clinical disease, and diagnostics," Vector Borne and Zoonotic Diseases, vol. 15, no. 4, pp. 223-230, 2014.

[15] A. F. Yusoff, A. N. Mustafa, H. M. Husaain et al., "The assessment of risk factors for the Central/East African genotype of Chikungunya Virus infections in the state of Kelantan: a case control study in Malaysia," BMC Infectious Diseases, vol. 13, no. 1, pp. 1-8, 2013.

[16] D. C. Kajeguka, R. D. Kaaya, S. Mwakalinga et al., "Prevalence of dengue and chikungunya virus infections in north-eastern Tanzania: a cross sectional study among participants presenting with malaria-like symptoms," BMC Infectious Diseases, vol. 16, no. 1, pp. 1-9, 2016.

[17] United States Center for Disease Control and Prevention and Pan American Health Organization, Preparedness and Response for Chikungunya Virus: Introduction in the Americas, https://iris.paho.org/bitstream/handle/10665.2/4009/
Chikungunya\%20Virus.pdf? sequence $=1$ \&isAllowed=yAccessed on 6 July, 2019.

[18] R. Balasubramanian, B. Anukumar, and L. Nikhil, "Stegomyia indices of Aedes Mosquito infestation and container productivity in Alappuzha district Kerala," International Journal of Mosquito Research, vol. 2, no. 2, pp. 14-18, 2015.

[19] V. Ramachandran, P. Manickam, P. Kaur et al., "Behavioural determinants associated with CHIKV outbreak in gouriepet, avadi, Chennai, south India," Journal of Biomedical Science, vol. 4 , p. 1, 2015.

[20] L. H. Degife, Y. Worku, D. Belay et al., "Factors associated with dengue fever outbreak in Dire Dawa administration city, October, 2015, Ethiopia - case control study," BMC Public Health, vol. 19, p. 650, 2019.

[21] F. Y. Sitep, A. Suprayogi, D. Pramono, H. Harapan, and M. Mudatsir, "Epidemiological investigation of chikungunya outbreak, West Kalimantan, Indonesia," Clinical Epidemiology and Global Health, vol. 8, pp. 113-116, 2020.

[22] A. Fred, A. Fianu, M. Béral et al., "Individual and contextual risk factors for chikungunya virus infection: the SEROCHIK cross-sectional population-based study," Epidemiology and Infection, vol. 146, no. 8, pp. 1056-1064, 2018. 